\title{
A Focused Small-Molecule Screen Identifies PHA-680626 as an Amphosteric Inhibitor Disrupting the Interaction between Aurora-A and N-Myc
}

Fani Souvalidou ${ }^{1^{*}}$, Dalila Boi ${ }^{1^{*}}$, Roberta Montanari ${ }^{2 *}$, Federica Polverino ${ }^{3}$, Grazia Marini ${ }^{1}$, Davide Capelli ${ }^{2}$, Giorgio Pochetti ${ }^{2}$, Roberto Contestabile ${ }^{1}$, Daniela Trisciuoglio ${ }^{3}$, Angela Tramonti ${ }^{3 \#}$, Patrizia Carpinelli ${ }^{4}$, Camilla Ascanelli ${ }^{5}$, Catherine Lindon ${ }^{5}$, Alessandro De Leo ${ }^{6}$, Roberto Di Santo ${ }^{6}$, Roberta Costi ${ }^{6}$, Giulia Guarguaglini ${ }^{3 \#}$, Alessandro Paiardini ${ }^{1 \#}$.

1. Department of Biochemical Sciences, Sapienza University, P. le Aldo Moro 5, 00185 Rome, Italy.

2. Istituto di Cristallografia, Consiglio Nazionale delle Ricerche, 00015 Monterotondo, Rome, Italy.

3. Istituto di Biologia e Patologia Molecolari, Consiglio Nazionale delle Ricerche, 00185 Rome, Italy.

4. Nerviano Medical Sciences S.r.l., Nerviano, Italy.

5. Department of Pharmacology, University of Cambridge, Tennis Court Road, Cambridge, CB2 1PD, UK.

6. Istituto Pasteur-Fondazione Cenci Bolognetti, Dipartimento di Chimica e Tecnologie del Farmaco, Sapienza University, P. le Aldo Moro 5, 00185 Rome, Italy.

\section{Corresponding Authors}

Alessandro Paiardini, alessandro.paiardini@uniroma1.it

Giulia Guarguaglini, giulia.guarguaglini@uniroma1.it

* These co-first authors contributed equally to the work

\# These co-last authors contributed equally to the work

\begin{abstract}
Neuroblastoma is a severe childhood disease, accounting for $10 \%$ of all infant cancers. The amplification of the MYCN gene, coding for the N-Myc transcriptional factor, is an essential marker correlated with tumor progression and poor prognosis. In neuroblastoma cells, the mitotic kinase Aurora-A (AURKA), also frequently overexpressed in cancer, prevents N-Myc degradation, by directly binding to a highly conserved N-Myc region, i.e. Myc Box I. As a result, elevated levels of N-Myc, which are required for the growth of MYCN amplified cells, are observed. During the last years, it has been demonstrated that the ATP competitive inhibitors of AURKA CD532, MLN8054 and Alisertib also cause essential conformational changes in the structure of the activation loop of the kinase that prevent $\mathrm{N}-\mathrm{Myc}$ binding, thus impairing the formation of the AURKA/N-Myc complex. In this study, starting from a screening of crystal structures of AURKA in complex with known inhibitors, we identified additional compounds affecting the conformation of the kinase activation loop. We assessed the ability of such compounds to disrupt the interaction between AURKA and N-Myc in vitro, using Surface Plasmon Resonance competition assays, and in tumor cell lines overexpressing MYCN, by performing Proximity Ligation Assays. Finally, their effects on N-Myc cellular levels and cell
\end{abstract}


viability were investigated. Our results, identifying PHA-680626 as an amphosteric inhibitor both in vitro and MYCN overexpressing cell lines, expand the repertoire of known conformational disrupting inhibitors of the AURKA/N-Myc complex, and confirm that altering the conformation of the activation loop of AURKA with a small molecule is an effective strategy to destabilize the AURKA/N-Myc interaction in neuroblastoma cancer cells.

\section{INTRODUCTION}

AURKA, a member of the Aurora Kinases family, is a Ser/Thr kinase involved in the progression of mitosis. In interphase it associates with centrosomes and controls their maturation in the G2 stage of the cell cycle, while in mitotic cells it localizes at spindle poles, where it plays a primary role in establishing spindle bipolarity and regulating microtubule nucleation and dynamic properties (Joukov \& De Nicolo, 2018; Magnaghi-Jaulin et al., 2019). AURKA is widely overexpressed in a variety of solid tumors (Bischoff et al., 1998; Sen et al., 2002; Du et al., 2021). In particular, in neuroblastoma, a severe childhood cancer that arises from highly proliferative migratory cells of the neural crest (Grimmer \& Weiss, 2006), AURKA is not only highly expressed relative to normal tissues (Zhou et al., 1998), but also displays another critical function, by binding to and stabilizing the oncoprotein N-Myc (Otto et al., 2009).

$\mathrm{N}-\mathrm{Myc}$ belongs to the MYC family together with C-Myc and L-Myc, a very potent network of transcriptional factors, regulating the expression of a massive group of genes implicated in cell cycle progression, protein translation and metabolism (Albihn et al., 2010; Grandori et al., 2000). Such transcriptional factors are usually found upregulated in various cancers in humans (Dang, 2012). The structural organization of Myc proteins shares conserved motifs called "Myc Boxes" (MB), which serve as a docking site for protein-protein interactions. The turnover of Myc proteins is regulated by the phosphorylation of $\mathrm{MBI}$ (Myc Box I), which directs the protein towards ubiquitination and proteolysis (Welcker et al., 2004). N-Myc is primarily phosphorylated on Ser62 by the Cdk1/Cyclin-B complex, and then also on Thr58 by the Gsk3 kinase (Yada et al., 2004; Sjostrom et al., 2005; Chesler et al., 2006). Dephosphorylation of Ser62 by the PP2A phosphatase recruits the activity of E3 ubiquitin ligase SCFFbxW7 (Welcker et al., 2004; Adhikary \& Eilers, 2005). The physical interaction of N-Myc with AURKA, as demonstrated by in vitro and crystallographic studies, does not allow the ubiquitin ligase to intervene, thus preventing N-Myc degradation (Richards et al., 2016).

During the years, serious efforts have been taken to exploit specific methods for targeting AURKA in cancer, mainly focused on the design of various inhibitors that are under investigation in clinical trials (Malumbres \& Ignacio, 2014; Levinson, 2018). Apparently, an important subclass of these inhibitors, hailed as "conformation disrupting" (CD) or "amphosteric", is also able to effectively disrupt the protein-protein interaction of the kinase with N-Myc, promoting the degradation of N-Myc itself (Gustafson et al., 2014; Meyerowitz et al., 2015; Brockmann et al., 2015). The binding of such inhibitors induces a series of unique interactions that produce a conformational change on the structure of the kinase domain of AURKA, distinct from any known physiological state (Meyerowitz et al., 2015). Notable examples come from CD532, MLN8054 or MLN8237 (Alisertib), diaminopyrimidine derived 
compounds that disrupt the interaction between AURKA and N-Myc, due to a significant shift of the $\mathrm{N}$-terminal domain relative to the C-terminal one, and most importantly to the repositioning of the kinase activation loop (the "A-loop", comprising residues 276-291 of human AURKA) in an inactive (hereinafter "closed") conformation, which prevents N-Myc binding (Gustafson et al., 2014; Richards et al., 2016).

Beside their classification as "orthosteric" (inhibiting kinase activity), "allosteric" (disrupting protein-protein interactions) or "amphosteric" (both), in general kinase inhibitors are classified as "type I" or "type II", according to their preference for a "DFG-out" or "DFG-in" target state, which refers in turn to the orientation of a catalytically important motif of three residues, i.e. Asp(D)-Phe(F)-Gly(G), found at the N-terminus of the kinase A-loop (Schindler et al., 2000; Pargellis et al., 2002; Wan et al., 2004). In the past, the closed state of the A-loop was invariably linked to the "DFG-out"-associated, type II inhibitors. However, recent studies have demonstrated that this is not a strict rule. For example, CD532, which is known to confer the closure of the A-loop, keeps the motif in state "DFG-in". Instead, a highly potent aminopyrimidinyl quinazoline AURKA type II inhibitor is not able to stabilize the A-loop in a closed state (Heron et al., 2006; PDB Code: 2C6E), as well as the potent MK8745 type-II compound, which does not act as a CD inhibitor (Lake et al., 2018).

Here we investigated, by computational and experimental means, which compounds already known to target AURKA at the ATP binding site (i.e., "orthosteric" compounds), regardless of their "type I" or "type II" nature, are able to act as conformation disrupting inhibitors, and destabilize the complex with N-Myc. An in-silico data mining in PDB of all known AURKA inhibitors was conducted, to investigate which molecules are capable of opening the "angle" of the kinase in a similar way to other CD inhibitors, as well as to close the A-loop. A series of identified compounds potentially acting as CD inhibitors were firstly confirmed with Surface Plasmon Resonance (SPR) and kinase activity assays to bind to AURKA at the active site and to inhibit its enzymatic activity. Using SPR competition assays with N-Myc, we demonstrate that some of those compounds so far known only as inhibitors of the kinase activity of AURKA, are indeed able to disrupt also the Aurora-A/N-Myc complex in vitro. Finally, those compounds were also tested in MYCN overexpressing cell lines, for their ability to disrupt the complex. The most important candidates, together with CD532, MLN8054 and Alisertib, represent new potential pharmacological tools to disrupt the "unholy matrimony" between two proteins that are mainly responsible for the severity and poor outcome of neuroblastoma.

\section{RESULTS}

\section{PHA-680626 and RPM1722 are predicted to prevent N-Myc binding to AURKA}

By screening the whole Protein Data Bank (PDB) subset of AURKA in complex with small molecule compounds, we initially superposed each structure with apo-AURKA (PDB: 4J8N) and with the AURKA/N-Myc complex (PDB: 5G1X) (Richards et al., 2016), and identified several entries in which both of the following conditions were satisfied: 1) AURKA opens its N-terminal domain relative to the $\mathrm{C}$-terminal one (the opening was assessed by measuring the angle between the $\alpha$-carbons of Val324, Glu308, and Ala172, as already described in Meyerowitz et 
al., 2015); 2) the A-loop of AURKA is in a state which is incompatible with N-Myc binding, due to the presence of active site inhibitors. Among these, we selected CD532 and MLN8054, which were already shown to decrease the amount of N-Myc in neuroblastoma cell lines (Brockmann et al., 2013; Gustafson et al., 2014 ) (Figure 1). Alisertib (MLN8237), whose structure in complex with AURKA has not been solved yet, was also added for its very high similarity with MLN8054. Moreover, the effects of CD532, MLN8054 and Alisertib have been already attributed to specific conformational changes of the kinase. Two additional compounds, i.e. PHA680626 and RPM1722 (Fancelli et al., 2006; Martin et al., 2012), already known for being orthosteric inhibitors of AURKA, but not investigated, to our knowledge, for their conformation disrupting potential, were selected for further investigation, since the opening of the kinase lobe was particularly high $\left(\geq 85^{\circ}\right)$, and the A-loop was stabilized in a closed conformation (Figure 1).

PHA-680626 derives from the optimization of PHA-680632 (Soncini et al., 2006), a compound based on a 1,4,5,6-tetrahydropyrrolo[3,4-c]pyrazole bi-cycle scaffold, which is known to target the ATP-binding pocket of kinases. The 5-amidothiophene substituent of PHA-680626 is directed away from the glycine-rich loop and packs around Leu263 and Ala273, in proximity of the DFG-motif. A key interaction stabilizing the A-loop in its closed state involves the stacking between the thiophene ring of PHA-680626 and His280 of the A-loop (Fancelli et al., 2006). RPM1722 relies on the bis-anilinopyrimidinic scaffold that is typical of several "DFG-in" inhibitors; however, a bromine substituent on the benzene ring of the scaffold, directed at the DFG flanking residue Ala273, is responsible for induced-dipole forces along alanine side chain, which are reflected by drastic conformational changes of the A-loop. This unique "DFGout/loop-in" conformation is also stabilized by hydrogen bonding interactions between RPM1722 and residues Lys141 and His280, and by a conformational shift of Trp277, moving from a polar environment to a hydrophobic pocket (Martin et al., 2012).

These observations confirm that other molecules, in addition to the already known amphosteric AURKA inhibitors, induce conformational changes at the kinase lobes and a complete flip of the A-loop, retaining AURKA in a closed conformation that could prevent $\mathrm{N}$ Myc binding.

\section{PHA-680626 and RPM1722 behave as CD inhibitors in vitro}

In order to verify that PHA-680626 and RPM1722, and the previously identified inhibitors (CD532, Alisertib, and MLN-8054), were active, in vitro kinase activity assays on the purified kinase domain of AURKA were carried out (Table 1 and Supplementary Figure 1). Ten different concentrations of each compound (solubilized in 100\% DMSO) were used in the presence of a concentration of ATP equal to the $K_{M}$ reported in the literature (Dodson \& Bayliss, 2012)(see Materials and Methods). The measured $\mathrm{IC}_{50}$ values were comparable to those found in literature (Martin et al., 2012; Gustafson et al., 2014).

In parallel, we also evaluated the interaction between the inhibitors and AURKA by Surface Plasmon Resonance (SPR) binding kinetic assay. AURKA was immobilized onto a PCH sensor chip (see Materials and Methods) and each compound was injected at increasing concentrations in running buffer (10 mM Hepes pH 7.4, $150 \mathrm{mM} \mathrm{NaCl}, 1 \%$ DMSO, 0.005\% 
Tween 20). The interaction between AURKA and the ligands was indicated by the increase of Resonance Units (RUs) compared to the baseline. As the dynamic equilibrium of the A-loop conformation of AURKA influences the binding of small molecule inhibitors, in order to minimize the low affinity binding mode, we carried out the experiment at a fast flow rate $(150 \mu \mathrm{l} / \mathrm{min})$ and, again, obtained for all compounds $\mathrm{Kd}$ values in agreement with data from literature (Table 2 and Figure 2A-E). An SPR binding kinetic assay was then used to evaluate the binding of the AURKA interacting region (AIR) of N-Myc (N-Myc residues 61-89) (Richards et al., 2016) to AURKA at the same conditions of flow rate and running buffer as for the inhibitors. A Kd value of $\sim 1 \mu \mathrm{M}$ was obtained (Figure $2 \mathrm{~F}$ ). After determining the affinity constants, we performed SPR competition experiments to evaluate the conformational disrupting potency of the inhibitors and their ability to interfere with N-Myc binding to the kinase. In particular, a saturating concentration of each inhibitor (at least 10 times higher than the Kd) was first added to the running buffer to ensure that all the sites of AURKA were filled. Then, N-Myc was injected at different concentrations (125 nM, $250 \mathrm{nM}, 500 \mathrm{nM}, 1 \mu \mathrm{M}, 2 \mu \mathrm{M}$, $4 \mu \mathrm{M}, 8 \mu \mathrm{M}, 16 \mu \mathrm{M})$ at a constant flow rate $(150 \mu \mathrm{l} / \mathrm{min})$ of running buffer. As a negative control, we also tested one of the most potent and specific inhibitors of AURKA, MK8745 (an analog of MK-5108 with $\mathrm{IC}_{50}$ of $0.6 \mathrm{nM}$ ) and ZM447439 (a pan-Aurora family inhibitor, with a measured I $C_{50}$ of $\sim 100$ nM) (Georgieva et al., 2010; Chowdhury et al., 2012). Results in Table 2 indicate that the selected inhibitors almost completely prevent N-Myc from binding to AURKA ( $\mathrm{Kd}_{\mathrm{N}-\mathrm{Myc}}>15 \mu \mathrm{M}$ or not measurable), thus demonstrating their behavior as conformation disrupting compounds, and confirming our initial hypothesis. In agreement with the structural analysis, MK8745 and ZM447439 do not act as CD inhibitors and they do not interfere with the AURKA/ N-Myc interaction (Table 2 and Figure 3).

\section{PHA-680626 behaves as a CD inhibitor in MYCN overexpressing cell lines}

We then wanted to test whether PHA-680626 and RPM1722 were able to act as CD-inhibitors, and thus to inhibit the N-Myc/AURKA interaction, in cultured cells. Since the interaction is reported as cell cycle-dependent (Buchel et al., 2017) and may vary depending on the levels of the two proteins in cancer cells, we employed a U2OS cell line, that does not express endogenous $\mathrm{N}-\mathrm{Myc}$, engineered for inducible doxycycline-dependent expression of mNeontagged N-Myc (Figure 4). Cultures treated with $1 \mu \mathrm{M}$ of each compound and with doxycycline were synchronized in the late $\mathrm{G} 2$ phase, when AURKA levels are highest, by the RO-3360 Cdk1 inhibitor (protocol in Figure 4A; Supplementary Figure 3A). Interestingly, N-Myc failed to accumulate to levels observed in control cultures (DMSO) after treatment with PHA-680626, suggesting an impaired interaction with AURKA that destabilizes it (Figure 4B). To directly measure the interaction between AURKA and N-Myc under these conditions, in situ Proximity Ligation assay reactions were set up (Figure $4 \mathrm{C}$ and Supplementary Figure 3B), adding the proteasome inhibitor MG-132 in the last 4 hours before fixation to counteract potential destabilization effects. After treatment with small molecule inhibitors isPLA signals were counted within cell nuclei and compared to control cells. Results indicate that cells with more numerous isPLA interaction spots are less represented in PHA-680626-treated cultures, to a higher extent compared to MLN8054, while RPM1722 is ineffective (Figure 4C.). This is 
accompanied by an increase in the percentage of cells with low isPLA signals after PHA-680626 treatment, indicating an impaired AURKA/N-Myc interaction. We then moved to a neuroblastoma MYCN-amplified cell line, i.e., IMR-32. Treatment for 48 hours with $1 \mu \mathrm{M}$ PHA680626 decreased N-Myc levels in a comparable manner to MLN8054; no major effect was observed with RPM1722 treatment (Figure 5A). By FACS analysis PHA-680626 yielded an increase in the fraction of cells with G2/M DNA content, similarly to known AURKA inhibitors (i.e., MLN8054, Figure 5B), while RPM1722 did not induce major cell cycle changes. Interestingly, in contrast to RPM1722, PHA-680626 administration affected IMR-32 cells morphology (Figure $5 \mathrm{C}$ ), with the disappearance of viable substrate-adherent cells and the formation of cellular aggregates that detach from the surface. Consistent with induction of cell death, PHA-680626 and MLN8054 treated cultures displayed a fraction of cells with sub-G1 DNA content (about $40 \%$ and $30 \%$ respectively, compared to $8 \%$ and $15 \%$ in DMSO or RPM1722 conditions), as well as a fraction of cleaved PARP-1 in western blot analysis (Figure $5 \mathrm{~A}$ and $5 \mathrm{~B})$.

Together, these results indicate that PHA-680626 is an effective candidate to prevent the AURKA/N-Myc interaction in cultured cells, triggers N-Myc degradation and impairs the viability of neuroblastoma cell lines that are N-Myc-addicted for their proliferation.

\section{DISCUSSION}

Poor prognosis for neuroblastoma patients is often associated to elevated levels of N-Myc, a transcriptional factor that mainly acts in favor of cellular proliferation, metastasis, cell cycle progression and protein translation (Dang, 2012). In spite of the paramount importance of this oncoprotein, the very low druggability of the latter has so far prevented any successful strategy involving $\mathrm{N}-\mathrm{Myc}$ as a potential target for chemotherapeutic intervention. However, recent studies demonstrating that the physical interaction between the mitotic kinase AURKA and NMyc sequesters the latter from proteolytic degradation (Richards et al., 2016; Büchel et al., 2017) paved the way for a new and promising chemotherapeutic approach in neuroblastoma. These findings have prompted the identification of compounds able to disrupt the AURKA/NMyc complex, in order to promote the degradation of the latter (Meyerowitz et al., 2015; Brockmann et al., 2015)(Gustafson et al., 2014). Already known competitive inhibitors of AURKA, targeting the kinase active site, were demonstrated to also inhibit such interaction. Among these, CD532, MLN8054 and Alisertib, the best characterized compounds so far, are able to alter the conformation of AURKA in a way that prevents N-Myc binding, and for this reason, they have been hailed as "conformation disrupting" (CD) or "amphosteric" inhibitors (Meyerowitz et al., 2015).

As the amphosteric effects are neglected in most current inhibitor screenings (Gustafson et al., 2014), we investigated already known AURKA inhibitors with the aim of 1) expanding the repertoire of AURKA/N-Myc amphosteric compounds, and 2) at the same time, providing additional insights for their observed chemotherapeutic effects. To this end, we started this study from structural data mining on PDB, to identify crystal structures of AURKA whose conformation, due to the complex with active site inhibitors, was unsuited for N-Myc binding. 
The comparison of such AURKA structures with apo-AURKA (PDB: 4J8N), showed that the Nand $\mathrm{C}$-terminal lobes of the kinase domain can adopt a very diverse set of orientations, ranging from $\sim 85^{\circ}$ in the case of MLN8054, to $\sim 93^{\circ}$ for CD532, and different conformations of the Aloop in the closed state, confirming that the view of kinases existing mainly in two states, "active" or "inactive", based on the DFG-motif orientation (DFG-in -> active, DFG-out -> inactive) is somewhat simplistic. For example CD532, the best characterized amphosteric inhibitor so far, and the one exerting at the same time the amplest opening of the AURKA lobes and the most dramatic effects on N-Myc degradation, is paradoxically also a "DFG-in" inhibitor, according to the position of the DFG motif (Meyerowitz et al., 2015).

Those compounds that were predicted to disrupt the AURKA conformation, in a way similar to MLN8054/CD532, were obtained and further characterized. A series of initial kinase activity assays confirmed the inhibitory ability of each compound against AURKA, with IC 50 in close proximity to the ones reported in literature with different in vitro/in vivo assays (Martin et al., 2012; Gustafson et al., 2014; Ferguson et al., 2017). MLN8054 (highly similar to Alisertib), with a 1-digit nanomolar IC 50 value, was the most potent AURKA inhibitor tested, and it was adopted as archetypal amphosteric inhibitor in the next comparative analyses.

Using SPR, a well-established technique that yields a straightforward characterization of protein-protein and protein-ligand interactions, we confirmed in vitro that the two compounds identified by structural analysis as potential amphosteric inhibitors, are indeed able to interfere to with AURKA/N-Myc formation. In one case (i.e., Alisertib), where it was still possible to measure an apparent $\mathrm{Kd}_{\mathrm{N}-\mathrm{Myc}}$, the observed effect was to lower the affinity of N-Myc for the kinase. All the other investigated compounds were able to completely inhibit the interaction between the two proteins at saturating concentrations (10 times higher than their Kd). PHA680626 was responsible for a complete dissociation of the complex. A closer inspection of the crystal structure and a detailed comparison of PHA-680626 with the highly similar PHA739358 (Danusertib), the structure of which was solved in complex with AURKA (PDB 2J50), provided a rationale to the different behavior of the two closely related compounds (Supplementary Figure 2A). Indeed, the thiophene moiety of PHA-680626 is able to stabilize the A-loop in a close conformation that is unproductive for N-Myc binding, thanks to a stacking interaction with His280 of the A-loop. Conversely, the bulkier methoxy moiety of Danusertib hampers such interaction and prevents the A-loop of AURKA to adopt a "closed" conformation. Notably, topologically similar hydrophobic interactions were also observed in the structure of AURKA/MLN8054 and predicted via modeling (Supplementary Figure 2B) in the case of the fluorophenyl moiety of Alisertib (residues Val147, Ser278 and Val279), thus suggesting such interaction as an important pharmacophore feature for stabilizing the closed conformation of the A-loop and conferring an amphosteric behavior to the inhibitor. It is interesting to note that CD532 achieves a similar conformational change of the A-loop, without engaging any stabilizing contact with the latter. Indeed, the deeply buried 3-trifluoromethylbiphenyl urea moiety of CD532 induces this inactive conformation via displacement and interactions with the $\beta 1$ and $\beta 2$ strands of the N-terminal lobe of AURKA, without reorienting the DFG motif, but wide-opening in this way the $\mathrm{N}$-terminal lobe of the kinase, creating a large cleft in which the A-loop is stabilized in an inactive orientation through a unique network of 
hydrogen bonds. Therefore, taken together with structural data, these observations suggest that the conformational disruption of AURKA that is necessary for dissociate N-Myc can be achieved by adopting two complementary, but distinct strategies: stabilizing the A-loop in a closed conformation via formation of direct contacts, and/or wide-opening the $\mathrm{N}$-terminal lobe of the kinase to create an ideal cleft for A-loop closure.

It is important to note that, in any case, fairly high concentrations of inhibitors were necessary to hamper the formation of the AURKA/N-Myc complex. Such high concentrations, which are necessary to unveil the amphosteric potential of AURKA inhibitors, suggest the difficulty that small inhibitors encounter when competing with the AIR of N-Myc. It was previously shown that AURKA is in dynamic equilibrium between active and inactive A-loop conformations and that the position of this equilibrium can be shifted by the binding of other ligands (Gilburt et al., 2017). The kinetics of N-Myc binding to AURKA (Figure 2F) measured by means of SPR suggests that an initial low association rate $\left(K_{o n}\right)$ is then followed by a quite stable complex that shifts the dynamic equilibrium of AURKA in a conformation disfavoring successive binding of $C D$ inhibitors. In this scenario, our data suggest that an amphosteric inhibitor of AURKA with a very low dissociation rate (in our study PHA-680626, for example, is the one with the lowest $\mathrm{K}_{\text {off }}$ ), which is independent on the concentration of the compound, might be more effective at displacing N-Myc. Recently, it has been shown that covalent Coenzyme A modification of AURKA via binding to the Cys290 residue of the A-loop is specific, and provides the proof of concept for a potential "dual anchor" irreversible inhibitory mechanism of AURKA (Tsuchiya et al., 2020). Such suicide inhibition would show the lowest reachable $K_{\text {off }}$ value and would possibly keep the A-loop of AURKA in a conformation unsuitable for N-Myc binding, relieving from the need of high inhibitor concentrations.

Most importantly, our study expanded the repertoire of AURKA/N-Myc amphosteric compounds and found that two other compounds other than CD532, Alisertib and MLN8054, i.e. PHA-680626 and RPM1722, already known as orthosteric inhibitors, are indeed able to prevent N-Myc binding to AURKA. When moving to cellular studies, PHA-680626 proved effective both in inducing a cell cycle arrest comparable to MLN8054 and in destabilizing NMyc. This was observed in a N-Myc inducible U2OS cell line and in neuroblastoma IMR32 cells, where cytotoxic effects were also observed. Surprisingly, no effect was induced by RPM1722 treatment, including no observable changes in the cell cycle phases distribution, which would be expected for an AURKA inhibitor; it is therefore possible that this compound does not efficiently enter the cell. To the best of our knowledge, no cell permeability studies were carried out so far on RPM1722. It is conceivable therefore that further optimization of this scaffold will be required, involving the improvement of cell permeability, by rational-based modifications of the original scaffold (e.g., carrier-linked prodrugs). On the other hand, the promising behavior of PHA-680626 suggests that its use in the treatment of neuroblastoma can be further explored. As discussed above, although the structural analysis of the highly similar Danusertib suggests that the latter is unable to exert the same effects of PHA-680626, nevertheless it will be interesting to investigate in future studies whether such "amphostericity" is a common attribute of this class of compounds. The recent report of successful combined use of AURKA CD-inhibitors and ATR inhibitors in MYCN amplified 
neuroblastoma (Roeschert et al., 2021), based on the S-phase function of the AURKA/N-Myc complex, strengthen the potential value of using compounds disrupting both AURKA activity and the interaction with $\mathrm{N}-\mathrm{Myc}$ for the treatment of this aggressive tumor type.

\section{MATERIALS AND METHODS}

\section{Screening on Protein Data Bank and Homology Modeling}

CD532 (PDB entry:4J8M) and MLN8054 (PDB entry: 2X81) were used as prototype of amphosteric inhibitors to quantitatively measure the magnitude of the conformational disrupting effect, as suggested in Meyerowitz et al., 2015. The opening between the N-terminal domain, compared to the C-terminal lobe, was asserted by measuring the dihedral angle between the $\alpha$-carbons of $\mathrm{V} 324, \mathrm{E} 308$, and $\mathrm{A} 172$, comparing it to the one measured for apoAURKA (empty binding pocket, 4J8N, angle: $81.7^{\circ}$ ) (Gustafson et al., 2014). In order to generate the ensemble of crystallographic structures for our analysis, we have conducted a data mining of all the available entries of AURKA kinase on Protein Data Bank (PDB). We have identified 198 structures for AURKA kinase overall and, in 95 of them, the kinase in complex with already known orthosteric inhibitors (March, 2018). Subsequently, we have conducted a visual examination of the identified structures using the open-source molecular visualization system PyMOL (The PyMOL Molecular Graphics System, Version 1.2r3pre, Schrödinger, LLC).

We proceeded aligning every protein-inhibitor complex with the PDB entries 4J8M and 2X81 in order to find other molecules that can both open the "angle" of Aurora-A and induce a complete flip of the activation loop, retaining it in a "closed" conformation not able to bind $\mathrm{N}$ Myc. We have excluded from our examination all the structures that lacked resolved residues at the key N-Myc binding regions; proceeding this way we have selected 10 entries (PDB codes: 2J4Z, 2J50, 2X6E, 2X81, 2WTV, 3UOJ, 3UOH, 3UNZ, 3UO6, 4J8M, 5ONE). We have then conducted another screening to exclude the "doublet" entries, in which Aurora-A is in complex with the same molecule (e.g., 2X81 and 2WTV in complex with MLN8054) and to consider only the best candidate in a family of inhibitors with the same backbone and the same structural change (e.g., 3UO6, 3UOJ, 3UOH, 3UNZ have the same chemical scaffold, but RPM1722 (3UOH) has the best affinity for AURKA, with a $\left.K_{d}=13 \pm 2.2 \mathrm{nM}\right)$. The final dataset was formed by four structures: 4J8M (Gustafson et al., 2014), 2X81 (Sloane et al., 2010), 2J4Z (Fancelli et al., 2006), $3 \mathrm{UOH}$ (Martin et al., 2012). The crystal structure of AURKA in complex with MLN8054 (PDB: 2X81) was used as a starting point to generate the model of AURKA in complex with Alisertib, using the "homology modeling" tool of PyMod 3.0 (Janson \& Paiardini, 2020).

\section{Purification of Aurora-A Catalytic Domain}

For the purification of the catalytic domain of human AURKA (amino acids 122-430), the plasmid PETM11 was used. The resulting protein contains an N-terminal His tag and an intervening TEV protease cleavage site. For the pre-inoculation, more colonies were taken from a plate and resuspended in LB containing $40 \mu \mathrm{g} / \mathrm{ml}$ kanamycin, with growth at $37^{\circ} \mathrm{C}$ overnight. After inoculation $1: 100$ in $2 \mathrm{~L}$ of $\mathrm{LB}$, culture was grown at $37^{\circ} \mathrm{C}$ for 4 hours and 30 minutes. When $\mathrm{OD}_{600 \mathrm{~nm}}$ achieved the value of 0.5 , the expression of the recombinant protein 
was induced with $0.2 \mathrm{mM} \mathrm{IPTG}$ and the culture was incubated at $28^{\circ} \mathrm{C}$ overnight. The pellet, after being centrifuged at $5500 \mathrm{rpm}$, at $4^{\circ} \mathrm{C}$ for $20 \mathrm{~min}$, was resuspended in about $130 \mathrm{ml}$ of 50 $\mathrm{mM}$ Hepes, $\mathrm{pH}$ 7.4, $5 \mathrm{mM} \mathrm{MgCl}$, $300 \mathrm{mM} \mathrm{NaCl}, 10 \%$ glycerol (buffer A) and 1 complete Protease Inhibitor Cocktail tablet (SIGMA-Aldrich), sonicated in ice (for $2 \mathrm{~min}, 20 \mathrm{sec}$ pulse on and $20 \mathrm{sec}$ pulse off) and centrifuged at $12000 \mathrm{rpm}$ for $25 \mathrm{~min}$ at $4^{\circ} \mathrm{C}$, twice. The obtained supernatant was filtered and loaded onto a 5-ml HisTrap column (GE Healthcare), previously equilibrated with buffer $A$ (flow rate $1 \mathrm{ml} / \mathrm{min}$ ). The column was washed with $10 \mathrm{ml}$ buffer $A$ and then with buffer A containing $20 \mathrm{mM}, 40 \mathrm{mM}$ and $100 \mathrm{mM}$ imidazole. Elution was performed with buffer A $+300 \mathrm{mM}$ imidazole. Fractions containing AURKA were collected and dialyzed against Buffer $A$, at $4^{\circ} \mathrm{C}$. In order to use the kinase for the activity assays, it was essential to phosphorylate the purified catalytic domain, so to activate it. That was accomplished by incubation of AURKA with $400 \mu \mathrm{M}$ of ATP ( $>10$ fold of $K_{m}$ ) for 3 hours on ice. To remove the ATP in excess, the protein was dialyzed in Buffer $A$ overnight at $4^{\circ} \mathrm{C}$. Once activated, AURKA was aliquoted and stored at $-80^{\circ} \mathrm{C}$, without losing stability or activity.

\section{Chemistry}

Compounds for testing were purchased from Molport (https://www.molport.com/). All chemicals were of the highest purity available (guaranteed purity of over $90 \%$ by H-NMR or LCMS), as certified by vendor. PHA-680626 was a gift by Nerviano Medical Sciences Srl. The synthesis of RPM1722 (RDS4007) was carried out as reported in Scheme 1 (see Supplementary Information), using the 2,4-dichloropyrimidine as starting material. Two subsequent aromatic nucleophilic substitutions were performed. First, the 2-bromoaniline was introduced in position 4 of the pyrimidine ring in the presence of aqueous hydrochloric acid (1.0 M), allowing the reaction to proceed for 36 hours at room temperature. Then, the second chlorine atom was substituted with 4-aminobenzoic acid, by means of a microwave-assisted reaction performed using absolute ethanol as a solvent at $100^{\circ} \mathrm{C}$ for 20 minutes. The synthesis was carried out providing some changes to a previously published work (Lawrence et al., 2012). The synthetic scheme, the procedures and the analytical spectroscopic data are reported in the Supplementary Information.

\section{Kinase Activity Assays}

All kinase assays for AURKA catalytic domain were performed with ADP-Glo ${ }^{\text {TM }}$ Kinase Assay Kit (Promega(C). For Alisertib the concentrations tested were: $0.075 \mathrm{nM}, 0.155 \mathrm{nM}, 0.312 \mathrm{nM}$, $0.665 \mathrm{nM}, 1.250 \mathrm{nM}, 2.5 \mathrm{nM}, 5 \mathrm{nM}, 10 \mathrm{nM}, 20 \mathrm{nM}$ and 40nM; for CD532: 3.12nM, 6.25nM, 12.5nM, 25nM, 50nM, 100nM, 200nM, 400nM, 800nM and 1600nM; for MLN8054: 0.15, 0.31, $0.62 \mathrm{nM}, 1.25 \mathrm{nM}, 2.5 \mathrm{nM}, 5 \mathrm{nM}, 10 \mathrm{nM}, 20 \mathrm{nM}, 40 \mathrm{nM}, 80 \mathrm{nM}$ and 160nM, for PHA680626: 3.12nM, 6.25nM, 12.5nM, 25nM, 50nM, 100nM, 200nM, 400nM, 800nM and 1600nM; for RMP1722 were: $0.5 \mathrm{nM}, 1 \mathrm{nM}, 2 \mathrm{nM}, 4 \mathrm{nM}, 8 \mathrm{nM}, 16 \mathrm{nM}, 32 \mathrm{nM}$ and $64 \mathrm{nM}$. The reaction mixture $(20 \mu \mathrm{l})$ contained the different concentrations of each inhibitor (obtained by adding $1 \mu \mathrm{l}$ of inhibitor solubilized in 100\% DMSO), $35 \mu \mathrm{M}$ ATP, 100 ng AURKA in $16 \mathrm{mM}$ Tris $\mathrm{HCl}, \mathrm{pH}$ 7.5, 8 $\mathrm{mM} \mathrm{MgCl} 2,0.04 \mathrm{mg} / \mathrm{ml} \mathrm{BSA}, 0.2 \mathrm{mM}$ DTT. After incubation at room temperature for $30 \mathrm{~min}$, the reaction was started by adding $5 \mu \mathrm{l}$ of Myelin Basic Protein $(20 \mathrm{mg} / \mathrm{ml})$, a substrate of 
AURKA and incubated at $30^{\circ} \mathrm{C}$. Then, the assay was performed in two steps; first, an equal volume $(25 \mu \mathrm{l})$ of ADP-Glo ${ }^{\text {TM }}$ Reagent was added to terminate the kinase reaction and deplete the remaining ATP. Second, the Kinase Detection Reagent ( $50 \mu \mathrm{l}$ ) was added to simultaneously convert ADP to ATP and allow the newly synthesized ATP to be measured using a luciferase/luciferin reaction. The luminescence produced at the end of every reaction, which corresponds to the kinase activity of AURKA, was measured as relative light unit (RLU) with a

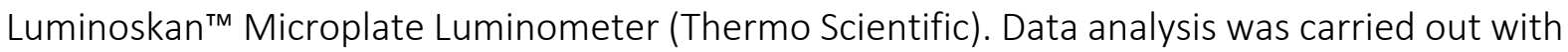
the PRISM8 (Graph Pad) software, using the following equation:

$$
R L U=100 \times\left(1-\frac{[I]^{n}}{[I]^{n}+I C 50^{n}}\right)+\text { const }
$$

from what we obtained the IC50, with [I] as the concentration of inhibitor, $n$ the Hill coefficient, and const as residual activity.

\section{Surface Plasmon Resonance}

SPR experiments were performed at $25{ }^{\circ} \mathrm{C}$ using a Pioneer AE optical biosensor (Molecular Devices-ForteBio) equipped with a PCH sensor chip and equilibrated with running buffer $10 \mathrm{mM}$ Hepes pH7.4, $150 \mathrm{mM} \mathrm{NaCl}, 1 \%$ DMSO, 0.005\% Tween20. Sensor chip was chemically activated for $7 \mathrm{~min}$ by injecting $175 \mu \mathrm{L}$ of a $1: 1$ mixture of $100 \mathrm{mM} \mathrm{N}$-hydroxysuccinimide (NHS) and 400 mM ethyl-3(3-dimethylamino) propyl carbodiimide (EDC) at a flow rate of 25 $\mu \mathrm{l} / \mathrm{min}$. AURKA was immobilized on both Ch1 and Ch3 channels of the activated sensor chip by using standard amine-coupling procedures (Lundström, 1994). Flow cell Ch2 was left empty and used as control. Briefly, a $0.1 \mathrm{mg} / \mathrm{mL}$ AURKA solution (in $10 \mathrm{mM}$ sodium acetate, $\mathrm{pH}$ 4.5) were injected at $10 \mu \mathrm{L} / \mathrm{min}$ on channels 1 and 3 (channel 2 was used as reference), followed by a $70-\mu \mathrm{L}$ injection of $1 \mathrm{M}$ ethanolamine $\mathrm{pH} 8.0$ to block any remaining activated groups on the surface. AURKA was captured to a density of $\sim 14000$ resonance units (RUs) on both Ch1 and Ch3 flow cells. The stability of the AURKA surface was demonstrated by the flat baseline achieved at the beginning (0-60s) of each sensorgram. To correct for bulk refractive index shifts, a DMSO calibration plot was also constructed (buffer sample containing 0.5-1.5\% DMSO) (Frostell-Karlsson et al., 2000).

Compounds for testing were solubilized in 100\% DMSO and then diluted in $10 \mathrm{mM}$ Hepes $\mathrm{pH}$ 7.4, $150 \mathrm{mM}$ sodium chloride and $0.005 \%$ Tween 20 up to a final concentration of $1 \%$ DMSO (running buffer) or 4\% (CD532) and injected at different concentrations onto the sensor chip at a constant flow rate of $150 \mu \mathrm{l} / \mathrm{min}$. A dissociation of $180 \mathrm{sec}$ was used and a mild regeneration of the surfaces was carried out every three injections by using a solution of $1 \mathrm{M} \mathrm{NaCl}$ and $10 \mathrm{mM} \mathrm{NaOH}$. At least two experiments for each analyte were performed. The interaction of the tested inhibitors with immobilized AURKA was detected as a measure of the change in mass concentration, expressed in RUs. The same conditions were used for the competition experiments between the compounds and N-Myc, respectively. In this case, N- 
Myc was diluted in running buffer containing the inhibitor at a constant concentration 10 times higher than its Kd. Different concentrations of N-Myc (ranging from $125 \mathrm{nM}$ to $16 \mu \mathrm{M}$ ) were then injected on the sensor chip at a constant flow of $150 \mu \mathrm{l} /$ min of running buffer containing the inhibitor at saturating concentration. All sensorgrams were processed by using double referencing (Myszka \& Morton, 1998). First, responses from the reference surface (Ch2) were subtracted from the binding responses collected over the reaction surfaces to correct for bulk refractive index changes between flow buffer and analyte sample. Second, the response from an average of the blank injections (zero analyte concentrations) was subtracted to compensate for drift and small differences between the active channel and the reference flow cell Ch2 (Myszka, 1999). To obtain kinetic rate constants and affinity constants, the corrected response data were fit in the QDAT software. A kinetic analysis of each ligand/analyte interaction was obtained by fitting the response data to a reversible 1:1 bimolecular interaction model. The equilibrium dissociation constant (Kd) was determined by the ratio $k_{\text {off }} / k_{\text {on }}$.

\section{Cell lines, synchronization protocols and treatments}

Cell lines were grown at $37^{\circ} \mathrm{C}$ and $5 \% \mathrm{CO}_{2}$. For the generation of the human U2OS/MYCNtFT osteosarcoma cell line, N-Myc (Addgene \#74163) was cloned into plasmid pcDNA5/FRT/TO (Thermo Fisher Scientific) bearing an mCherry and mNeon tandem fluorescent tag (tFT, Khmelinskii et al. 2012; kind gift of Michael Knop, ZMBH, University of Heidelberg). This construct was used to create the N-Myc-tFT cell line by Flp recombinasemediated integration into the genome of a U2OS FRT/TO Flp-In host cell line (kind gift of Adrian Saurin, Dundee) according to the manufacturer's instructions for the FIp-InTM TREXTM System (Thermo Fisher Scientific). This cell line was grown in complete DMEM (Dulbecco's Modified Eagle Medium) supplemented with 10\% tetracycline-free fetal bovine serum (FBS) and the induction of N-Myc expression was obtained by adding $1 \mu \mathrm{g} / \mathrm{ml}$ doxycycline (tetracycline analogue; Santa Cruz Biotechnology). Treatment with $9 \mu \mathrm{M}$ Ro3306 (Sigma-Aldrich, SML0569) for 22 h or with 10 MM MG132 (Chem-Cruz, sc-201270) for $4 \mathrm{~h}$ was performed when indicated. The IMR32 neuroblastoma cell line (kind gift of Prof. Giuseppe Giannini, Sapienza University of Rome) was grown in MEM (Minimum Essential Medium) supplemented with $10 \% \mathrm{FBS}$ and $1 \%$ of non-essential amino acids.

Both cell lines were treated with $1 \mu \mathrm{M}$ of inhibitors (MLN8054, PHA-680626, RPM1722), or $0,1 \%$ DMSO as control, for $22 \mathrm{~h}$ or $48 \mathrm{~h}$, as indicated. Images of IMR32 cultures after treatments were acquired using a ZOE fluorescent cell imager (Biorad).

\section{In Situ Proximity Ligation Assays (isPLA)}

In situ proximity ligation assays (isPLA) were performed on cells grown on coverslips and fixed with 3.7\% formaldehyde/30 mM sucrose in PBS, 10 min at room temperature, followed by permeabilization in PBS containing $0.1 \%$ TritonX-100, 5 min at room temperature. The Duolink PLA kit (DU092007, Sigma-Aldrich) was used according to manufacturer's instructions. The amplification time was 100 minutes and the primary antibodies pair to detect the interaction was rabbit anti-Aurora-A (14475, Cell Signaling, 1:100 o.n.) and 
mouse anti-N-Myc (NCM $\|$ 100, Calbiochem, 1:20). Counterstaining. with DAPI was performed at the end of the procedure. For quantification of isPLA fluorescence signals, samples were analyzed with an inverted microscope Eclipse Ti (Nikon) using a Clara camera (ANDOR technology) and 60x (oil immersion, N.A. 1.4) objective along the z-axis every 0.4 $\mu \mathrm{m}$ for a range of $3.2 \mu \mathrm{m}$. Images were acquired in automated mode with the JOBS module of the Nis-Elements H.C. 5.11 software. The "general analysis" module of Nis Elements H.C. 5.11 was then used for automatic spot count; isPLA signals were identified based on fixed parameters in all images and those within nuclei (defined by DAPI signal) were counted. Elaboration of the images was made by Adobe Photoshop CS 8.0 and statistical analyses were performed with GraphPad; specific tests are indicated in Figure legends.

\section{Western Blotting}

For Western Blot analysis, synchronized or asynchronously growing cells were lysed in RIPA buffer (50 mMTris-HCl pH 8.0, 150 mM NaCl, 1\% NP40, 1 mM EGTA, 1 mM EDTA, 0.25\% sodium deoxycholate) supplemented with protease and phosphatase inhibitors (Roche Diagnostic). Proteins were resolved by $10 \%$ SDS PAGE and transferred on a nitrocellulose membrane (Protran BA83, GE Healthcare) using a semi-dry system (Bio-Rad Laboratories S.r.I.). About 30 $\mu \mathrm{g}$ of extract per lane was loaded. Antibodies were: mouse anti-N-Myc (NCM II 100, Calbiochem, 1:100); rabbit anti-Lamin B1 (ab16048, Abcam, 1:4000); rabbit anti-PARP (9542, Cell Signaling, 1:1000 o.n.). HRP-conjugated secondary antibodies (BioRad) were revealed using the Clarity Western ECL substrate (BioRad, 170-5061).

\section{FACS analysis}

To analyze cell-cycle phases distribution, both non-adherent and adherent cells were collected by centrifugation, washed with PBS and fixed with $50 \%$ methanol overnight at $+4^{\circ} \mathrm{C}$. Cell cycle phase distribution was analyzed after incubation for 30 minutes in the dark with propidium iodide ( $\mathrm{Pl}, 0.03 \mathrm{mg} / \mathrm{ml}$ ) and RNAsi A $(0.2 \mathrm{mg} / \mathrm{ml})$ using a flow cytofluorimeter Epics XL apparatus (Beckman Coulter). Cell aggregates were gated out on bi-parametric graph FL-3lin/ratio as described in (Ferrara et al., 2018). Cell samples were analyzed in a Coulter Epics XL cytofluorometer (Beckman Coulter) equipped with EXPO 32 ADC software. At least 10000 cells per sample were acquired. The percentage of cells in the different phases of cell-cycle and in sub-G1 compartment was calculated using Flowing Software 2.5.1.

\section{Acknowledgments}

We thank I.A. Asteriti for experimental support and insightful discussions. The authors wish to thank Richard Bayliss and Dr. Mark Richards for assisting this study by providing the vector PETM6T1, coding for the 28-89 peptide of N-Myc, the vector pETM11 coding for Aurora-A Catalytic Domain (aa 122-403) as well as for their interesting advice, discussions and precious help. Thanks to Michael Knop for tFT plasmids and to Adrian Saurin for U2OS FRT/TO cells. This work is dedicated to the memory of our beloved mentors Prof. Francesco Bossa and Prof. Donatella Barra. 


\section{Funding Statement}

AP received support from Associazione Italiana Ricerca sul Cancro (AIRC, https://www.airc.it/) MFAG 20447 and Progetti Ateneo Sapienza University of Rome (https://www.uniroma1.it). AP acknowledges the CINECA award under the ISCRA initiative, for the availability of highperformance computing resources and support (IsC68_altmod). The funders had no role in study design, data collection and analysis, decision to publish, or preparation of the manuscript.

\section{REFERENCES}

Adhikary, S., Eilers, M. (2005). Transcriptional regulation and transformation by Myc proteins. Nat Rev Mol Cell, 6(8), 635-645.

Albihn, A., Johnsen, J. I., Henriksson, M. A. (2010). MYC in oncogenesis and as a target for cancer therapies. Advances in Cancer Research, 107(10), 163-224.

Bavetsias, V., Large, J. M., Sun, C., Bouloc, N., Kosmopoulou, M., Matteucci, M., Wilsher, N. E., Martins, V., Reynisson, J., Atrash, B., Faisal, A., Urban, F., Valenti, M., de Haven Brandon, A., Box, G., Raynaud, F. I., Workman, P., Eccles, S. A., Bayliss, R., Blagg, J., Linardopoulos, S., McDonald, E. (2010). Imidazo[4,5-b]pyridine Derivatives As Inhibitors of Aurora Kinases: Lead Optimization Studies toward the Identification of an Orally Bioavailable Preclinical Development Candidate. Journal of Medicinal Chemistry, 53(14), 5213-5228.

Bischoff, J. R., Anderson, L., Zhu, Y., Mossie, K., Ng, L., Souza, B., Schryver, B., Flanagan, P., Clairvoyant, F., Ginther, C., Chan, C. S. M., Novotny, M., Slamon, D. J., \& Plowman, G. D. (1998). A homologue of Drosophila aurora kinase is oncogenic and amplified in human colorectal cancers. EMBO J, 17(11), 3052-3065.

Brockmann, M., Poon, E., Berry, T., Carstensen, A., Deubzer, H. E., Rycak, L., Jamin, Y., Thway, K., Robinson, S. P., Roels, F., Witt, O., Fischer, M., Chesler, L., \& Eilers, M. (2013). Small Molecule Inhibitors of Aurora-A Induce Proteasomal Degradation of N-Myc in Childhood Neuroblastoma. Cancer Cell, 24(1), 75-89.

Büchel, G., Carstensen, A., Mak, K. Y., Roeschert, I., Leen, E., Sumara, O., Hofstetter, J., Herold, S., Kalb, J., Baluapuri, A., Poon, E., Kwok, C., Chesler, L., Maric, H. M., Rickman, D. S., Wolf, E., Bayliss, R., Walz, S., \& Eilers, M. (2017). Association with Aurora-A Controls N-MYC-Dependent Promoter Escape and Pause Release of RNA Polymerase II during the Cell Cycle. Cell Reports, 21(12), 3483-3497.

Chesler, L., Schlieve, C., Goldenberg, D. D., Kenney, A., Kim, G., McMillan, A., Matthay, K. K., Rowitch, D., \& Weiss, W. A. (2006). Inhibition of Phosphatidylinositol 3-Kinase Destabilizes 
Mycn Protein and Blocks Malignant Progression in Neuroblastoma. Cancer Res, 66(16) 81398146.

Chowdhury, A., Chowdhury, S., Tsai, M. Y. (2012). A novel Aurora kinase A inhibitor MK-8745 predicts TPX2 as a therapeutic biomarker in non-Hodgkin lymphoma cell lines. Leukemia \& Lymphoma, 53(3), 462-471.

Dang, C. V. (2012). Review MYC on the Path to Cancer. Cell, 149(1), 22-35.

Dodson, C. A., Bayliss, R. (2012). Activation of Aurora-A kinase by protein partner binding and phosphorylation are independent and synergistic. Journal of Biological Chemistry, 287(2), 1150-1157.

Du, R., Huang, C., Liu, K., Li, X., \& Dong, Z. (2021). Targeting AURKA in Cancer: molecular mechanisms and opportunities for Cancer therapy. Molecular Cancer, 20(1), 1-27.

Fancelli, D., Moll, J., Varasi, M., Bravo, R., Artico, R., Berta, D., Bindi, S., Cameron, A., Candiani, I., Cappella, P., Carpinelli, P., Croci, W., Forte, B., Giorgini, M. L., Klapwijk, J., Marsiglio, A., Pesenti, E., Rocchetti, M., Roletto, F., Severino, D., Soncini, C., Storici, P., Tonani, R., Zugnoni, P., Vianello, P. (2006). 1,4,5,6-Tetrahydropyrrolo[3,4-c]pyrazoles: Identification of a potent aurora kinase inhibitor with a favorable antitumor kinase inhibition profile. Journal of Medicinal Chemistry, 49(24), 7247-7251.

Ferrara, M., Sessa, G., Fiore, M., Bernard, F., Asteriti, I. A., Cundari, E., ... Degrassi, F. (2018). Small molecules targeted to the microtubule-Hec1 interaction inhibit cancer cell growth through microtubule stabilization. Oncogene, 37(2), 231-240.

Frostell-Karlsson, Å., Remaeus, A., Roos, H., Andersson, K., Borg, P., Hämäläinen, M., \& Karlsson, R. (2000). Biosensor analysis of the interaction between immobilized human serum albumin and drug compounds for prediction of human serum albumin binding levels. Journal of Medicinal Chemistry, 43(10), 1986-1992.

Georgieva, I., Koychev, D., Wang, Y., Holstein, J., Hopfenmüller, W., Zeitz, M., \& Grabowski, P. (2010). ZM447439, a novel promising aurora kinase inhibitor, provokes antiproliferative and proapoptotic effects alone and in combination with bio- and chemotherapeutic agents in gastroenteropancreatic neuroendocrine tumor cell lines. Neuroendocrinology, 91(2), 121-130.

Gilburt, J. A. H., Sarkar, H., Sheldrake, P., Blagg, J., Ying, L., \& Dodson, C. A. (2017). Dynamic Equilibrium of the Aurora A Kinase Activation Loop Revealed by Single-Molecule Spectroscopy. Angewandte Chemie - International Edition, 56(38), 11409-11414. 
Grandori, C., Cowley, S. M., James, L. P., Eisenman, R. N. (2000). The Myc/Max/Mad network and the transcriptional control of cell behavior. Annual Review of Cell and Developmental Biology, 16(January), 653-699.

Grimmer, M. R., \& Weiss, W. A. (2006). Childhood tumors of the nervous system as disorders of normal development. Current Opinion in Pediatrics, 18(6), 634-638.

Gustafson, W. C., Meyerowitz, J. G., Nekritz, E. A., Chen, J., Benes, C., Charron, E., Simonds, E. F., Seeger, R., Matthay, K. K., Hertz, N. T., Eilers, M., Shokat, K. M., \& Weiss, W. A. (2014). Drugging MYCN through an Allosteric Transition in Aurora Kinase A. Cancer Cell, 26(3), 414427.

Janson, G., \& Paiardini, A. (2020). PyMod 3: a complete suite for structural bioinformatics in PyMOL. Bioinformatics.

Joukov, V., \& De Nicolo, A. (2018). Aurora-PLK1 cascades as key signaling modules in the regulation of mitosis. Science Signaling, 11(543), 1-26.

Khmelinskii, A., Keller, P. J., Bartosik, A., Meurer, M., Barry, J. D., Mardin, B. R., Kaufmann, A., Trautmann, S., Wachsmuth, M., Pereira, G., Huber, W., Schiebel, E., Knop, M. (2012). Tandem fluorescent protein timers for in vivo analysis of protein dynamics. Nature Biotechnology, 30(7), 708-714.

Lake, E. W., Muretta, J. M., Thompson, A. R., Rasmussen, D. M., Majumdar, A., Faber, E. B., Ruff, E. F., Thomas, D. D., Levinson, N. M. (2018). Quantitative conformational profiling of kinase inhibitors reveals origins of selectivity for Aurora kinase activation states. Proceedings of the National Academy of Sciences, 115(51), E11894-E11903.

Lawrence, H.R., Martin, M.P., Luo, Y., Pireddu, R., Yang, H., Gevariya, H., Ozcan, S., Zhu, J., Kending, R., Rodriguez, M., Elias, R., Cheng, J.Q., Sebti, S.M., Schonbrunn, E., \& Lawrence, N.J. (2012). Development of o-Chlorophenyl substituted pyrimidines as exceptionally potent aurora kinase inhibitors. Journal of Medicinal Chemistry, 55(17), 7392-7416.

Levinson, N. M. (2018). The multifaceted allosteric regulation of Aurora kinase A. Biochemical Journal, 475(12), 2025-2042.

Lundström, I. (1994). Real-time biospecific interaction analysis. Biosensors and Bioelectronics, 9(9-10), 725-736.

Magnaghi-Jaulin, L., Eot-Houllier, G., Gallaud, E., \& Giet, R. (2019). Aurora A protein kinase: To the centrosome and beyond. Biomolecules, 9(1), 28. 
Malumbres, M., \& Ignacio, P. (2014). Aurora kinase A inhibitors: promising agents in antitumoral therapy. Expert Opin Ther Targets, 18(12), 1377-1393.

Martin, M. P., Zhu, J. Y., Lawrence, H. R., Pireddu, R., Luo, Y., Alam, R., Ozcan, S., Sebti, S. M., Lawrence, N. J., \& Schönbrunn, E. (2012). A novel mechanism by which small molecule inhibitors induce the DFG flip in Aurora A. ACS Chemical Biology, 7(4), 698-706.

Meyerowitz, J. G., Weiss, W. A., \& Gustafson, W. C. (2015). A new "angle" on kinase inhibitor design: Prioritizing amphosteric activity above kinase inhibition. Molecular and Cellular Oncology, 2(2), 10-12.

Myszka, D. G. (1999). Improving biosensor analysis. Journal of Molecular Recognition, 12(5), 279-284.

Myszka, D. G., \& Morton, T. A. (1998). Clamp: A biosensor kinetic data analysis program. Trends in Biochemical Sciences, 23(4), 149-150.

Otto, T., Horn, S., Brockmann, M., Eilers, U., Schüttrumpf, L., Popov, N., Kenney, A. M., Schulte, J. H., Beijersbergen, R., Christiansen, H., \& Berwanger, B., Eilers, M. (2009). Stabilization of NMyc Is a Critical Function of Aurora A in Human Neuroblastoma. Cancer Cell, 15(1), 67-78.

Pargellis, C., Tong, L., Churchill, L., Cirillo, P. F., Gilmore, T., Graham, A. G., Grob, P. M., Hickey, E. R., Moss, N., Pav, S., Regan, J. (2002). Inhibition of p38 MAP kinase by utilizing a novel allosteric binding site. Nature Structural Biology, 9(4), 268-272.

Richards, M. W., Burgess, S. G., Poon, E., Carstensen, A., Eilers, M., Chesler, L., Bayliss, R. (2016). Structural basis of N-Myc binding by Aurora-A and its destabilization by kinase inhibitors. Proceedings of the National Academy of Sciences of the United States of America, 113(48), 13726-13731.

Schindler, T., Bornmann, W., Pellicena, P., Miller, W. T., Clarkson, B., \& Kuriyan, J. (2000). Structural mechanism for STI-571 inhibition of Abelson tyrosine kinase. Science, 289(5486), 1938-1942.

Sen, S., Zhou, H., Zhang, R., Yoon, D. S., Vakar-lopez, F., Ito, S., Jiang, F., Johnston, D., Grossman, H. B., Ruifrok, A. C., Katz, R. L., Brinkley, W., \& Czerniak, B. (2002). Amplification / Overexpression of a Mitotic Kinase Gene in Human Bladder Cancer. Journal of the National Cancer Institute, 94(17), 1320-1329.

Sjostrom, S. K., Finn, G., Hahn, W. C., Rowitch, D. H., \& Kenney, A. M. (2005). The Cdk1 Complex Plays a Prime Role in Regulating N-Myc Phosphorylation and Turnover in Neural Precursors. Developmental Cell, 9(3), 327-338. 
Sloane, D. A., Trikic, M. Z., Chu, M. L. H., Lamers, M. B. A. C., Mason, C. S., Mueller, I., Savory, W. J., Williams, D. H., \& Eyers, P. A. (2010). Drug-Resistant Aurora A Mutants for Cellular Target Validation of the Small Molecule Kinase Inhibitors MLN8054 and MLN8237. ACS Chemical Biology, 5(6), 563-576.

Soncini, C., Carpinelli, P., Gianellini, L., Fancelli, D., Vianello, P., Rusconi, L., Storici, P., Zugnoni, P., Pesenti, E., Croci, V., Ceruti, R., Giorgini, M. L., Cappella, P., Ballinari, D., Sola, F., Varasi, M., Bravo, R., Moll, J. (2006). PHA-680632, a novel aurora kinase inhibitor with potent antitumoral activity. Clinical Cancer Research, 12(13), 4080-4089.

Tsuchiya, Y., Byrne, D. P., Burgess, S. G., Bormann, J., Baković, J., Huang, Y., Zhyvoloup, A., Yu, B. Y. K., Peak-Chew, S., Tran, T., Bellany, F., Tabor, A. B., Chan, A. E., Guruprasad, L., Garifulin, O., Filonenko, V., Vonderach, M., Ferries, S., Eyers, C. E., Carroll, J., Skehel, M., Bayliss, R., Eyers, P., Gout, I. (2020). Covalent Aurora A regulation by the metabolic integrator coenzyme A. Redox Biology, 28, 101318.

Wan, P. T. C., Garnett, M. J., Roe, S. M., Lee, S., Niculescu-Duvaz, D., Good, V. M., Project, C. G., Jones, C. M., Marshall, C. J., Springer, C. J., Barford, D., \& Marais, R. (2004). Mechanism of activation of the RAF-ERK signaling pathway by oncogenic mutations of B-RAF. Cell, 116(6), 855-867.

Welcker, M., Orian, A., Jin, J., Grim, J. A., Harper, J. W., Eisenman, R. N., \& Clurman, B. E. (2004). The Fbw7 tumor suppressor regulates glycogen synthase kinase 3 phosphorylation-dependent c-Myc protein degradation. Proceedings of the National Academy of Sciences of the United States of America, 101(24), 9085-9090.

Yada, M., Hatakeyama, S., Kamura, T., Nishiyama, M., Tsunematsu, R., Imaki, H., Ishida, N., Okumura, F., Nakayama, K., Nakayama, K. I. (2004). Phosphorylation-dependent degradation of c-Myc is mediated by the F-box protein Fbw7. EMBO Journal, 23(10), 2116-2125.

Zhou, H., Kuang, J., Zhong, L., Kuo, W., Gray, J. W., Sahin, A., Brinkley, B. R., Sen, S. (1998). Tumour amplified kinase STK15 / BTAK induces centrosome amplification, aneuploidy and transformation. Nature Genetics, 20(2), 189-193. 
bioRxiv preprint doi: https://doi.org/10.1101/2021.03.10.433854; this version posted April 29, 2021. The copyright holder for this preprint

(which was not certified by peer review) is the author/funder, who has granted bioRxiv a license to display the preprint in perpetuity. It is made available under aCC-BY-NC-ND 4.0 International license.

TABLES

Table 1. List of AURKA inhibitors investigated in this study*.

\begin{tabular}{llllll}
\hline PDB Code & Inhibitor & PDB ID & Selectivity & IC50 (nM) & Angle ( $\left.{ }^{\circ}\right)$ \\
\hline 4J8M & CD532 & CJ5 & AURKA & 35 & 92.9 \\
2 X81 & MLN8054 & ZZL & AURKA & 6 & 84.6 \\
$2 \mathrm{J4Z}$ & PHA-680626 & 626 & PAN & 99 & 88.9 \\
3 3UOH & RPM1722 & OC4 & AURKA & 19 & 85.5 \\
- & MLN8237* & A5B & AURKA & 6 & $85.0^{* *}$ \\
\hline
\end{tabular}

* Alisertib (IC $50=6 \mathrm{nM}$ ), whose structure in complex with AURKA has not been solved yet, was also added for its high similarity with MLN8054. ** Value computed from the homology model based on $2 \times 81$.

Table 2. SPR analyses of ligands binding. Full kinetic analysis of the AURKA inhibitors.

\begin{tabular}{llllll}
\hline Inhibitors & $\mathrm{K}_{\text {on }}\left(\mathrm{M}^{-1} \mathrm{~s}^{-1}\right)$ & $\mathrm{K}_{\text {off }}\left(\mathrm{s}^{-1}\right)$ & $\mathrm{Kd}(\mathrm{nM})$ & $\operatorname{Res} \mathrm{SD}$ & $\mathrm{Kd}_{\mathrm{N}-\mathrm{Myc}}(\mu \mathrm{M})$ \\
\hline CD532 & $3.58 \pm 0.05 \mathrm{e} 6$ & $0.0937 \pm 0.0007$ & $26.2 \pm 0.4$ & 0.83 & No binding \\
MLN8054 & $2.02 \pm 0.03 \mathrm{e} 7$ & $0.0461 \pm 0.0004$ & $2.28 \pm 0.03$ & 1.254 & No binding \\
PHA-680626 & $9.15 \pm 0.04 \mathrm{e} 5$ & $7.83 \pm 0.02 \mathrm{e}-3$ & $8.56 \pm 0.04$ & 3.7 & No binding \\
RPM1722 & $2.61 \pm 0.06 \mathrm{e} 6$ & $0.013 \pm 0.0001$ & $5.0 \pm 0.2$ & 3 & No binding \\
Alisertib & $8.2 \pm 0.1 \mathrm{e} 4$ & $5.04 \pm 0.04 \mathrm{e}-3$ & $60 \pm 1.0$ & 2.3 & 48 \\
\hline MK8754 & $7.8 \pm 0.1 \mathrm{e} 6$ & $0.01205 \pm 0.00009$ & $1.55 \pm 0.03$ & 1.65 & 0.7 \\
ZM447439 & $9.6 \pm 0.06 \mathrm{e} 4$ & $9.07 \pm 0.04 \mathrm{e}-3$ & $92 \pm 0.7$ & 1.09 & 4 \\
\hline
\end{tabular}


Figure 1

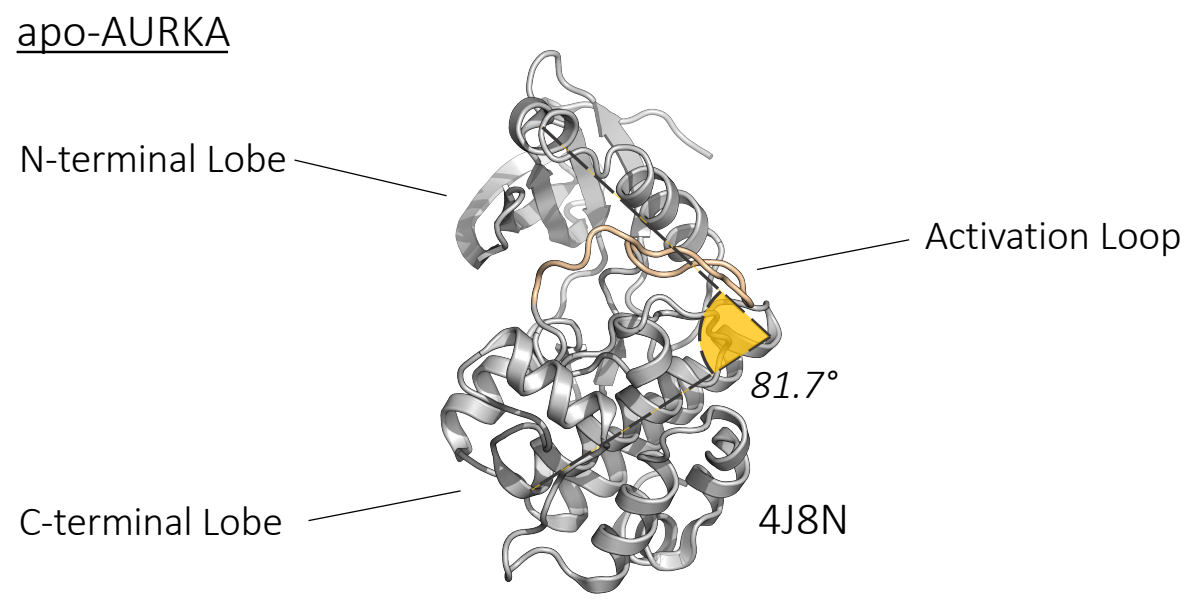

AURKA in complex with inhibitors
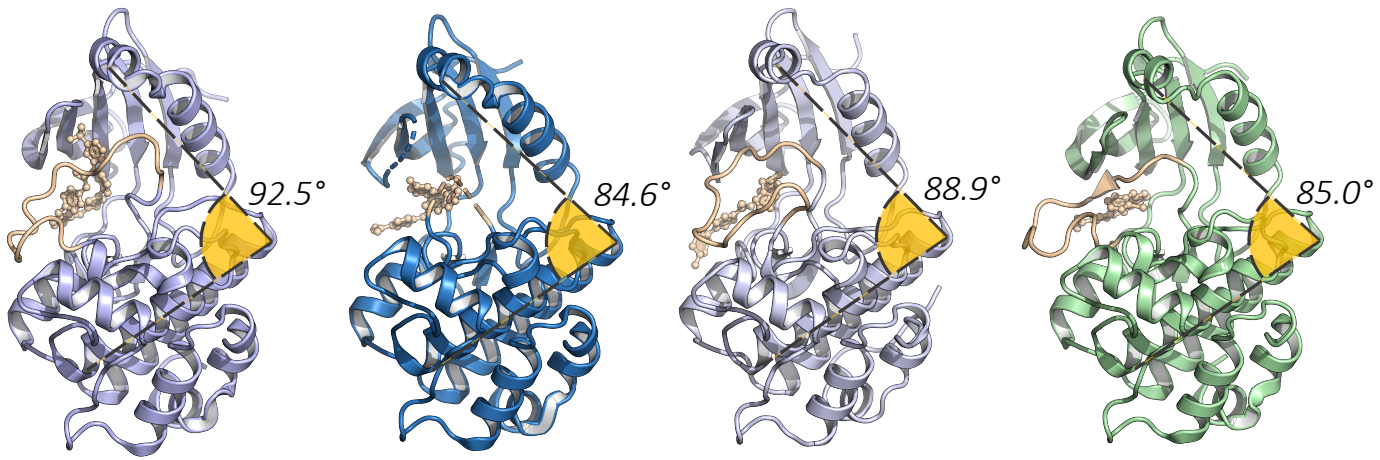

$4 \mathrm{~J} 8 \mathrm{M}-\mathrm{CD} 532$

2X81-MLN8054

2J4Z-PHA680626

3UOH-RPM1722
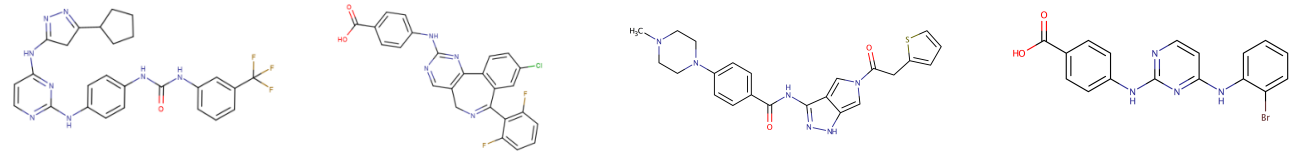

Figure 1. Structures of human AURKA in complex with the investigated inhibitors. The activation loop $(\mathrm{AL})$ is shown in orange. The angle between the $\mathrm{N}$ - and $\mathrm{C}$ - lobes, measured between $\alpha$-carbons of $\mathrm{V} 324, \mathrm{E} 308$, and $\mathrm{A} 172$, is also shown. The chemical structures of the compounds investigated is also represented. 
Figure 2
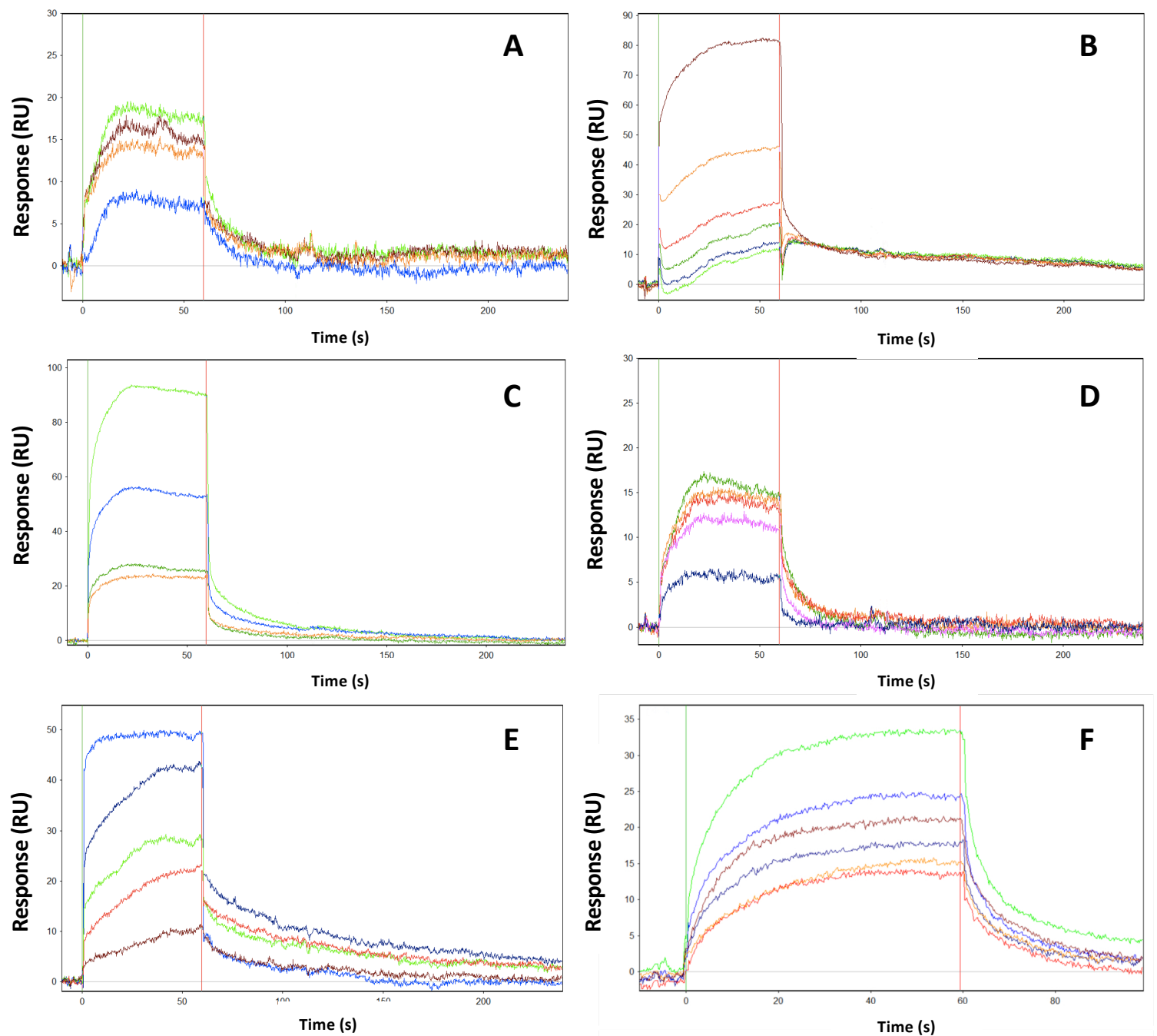

Figure 2. SPR analyses of ligands binding to AURKA. Full kinetic analysis of the AURKA inhibitors CD532 (A), MLN8054 (B), PHA-680626 (C), RPM1722 (D), Alisertib (E), and N-Myc (F). Determined binding parameters are listed in Table 2. Kinetic data for N-Myc: $\mathrm{K}_{\mathrm{on}}, 6.3 \pm 1 \mathrm{e} 4 \mathrm{M}^{-}$ ${ }^{1} \mathrm{~s}^{-1} ; \mathrm{K}_{\mathrm{off}}, 0.0659 \pm 0.0008 \mathrm{~s}^{-1} ; \mathrm{Kd}, 990 \mathrm{nM}$; Res SD 2.17. The interaction between AURKA and the ligands is indicated by the increase of Resonance Units (RUs) on the vertical axis compared to the baseline. 


\section{Figure 3}
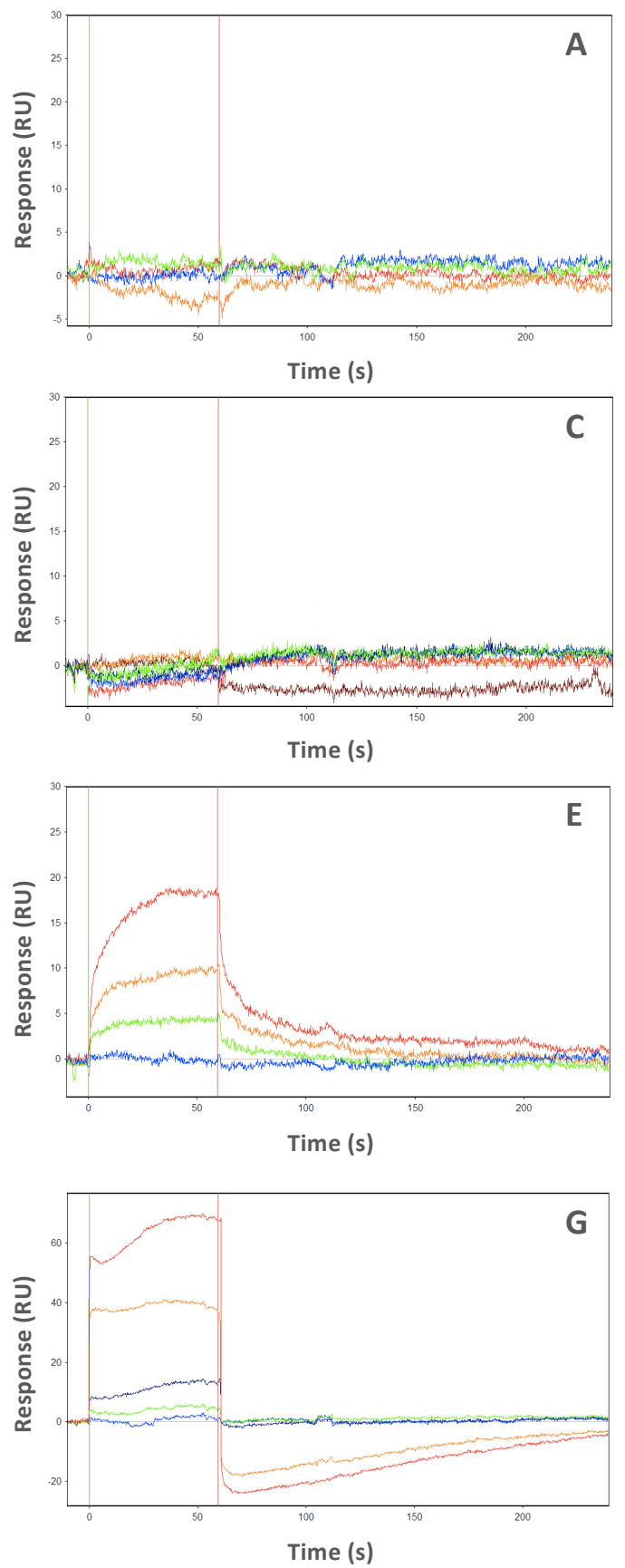
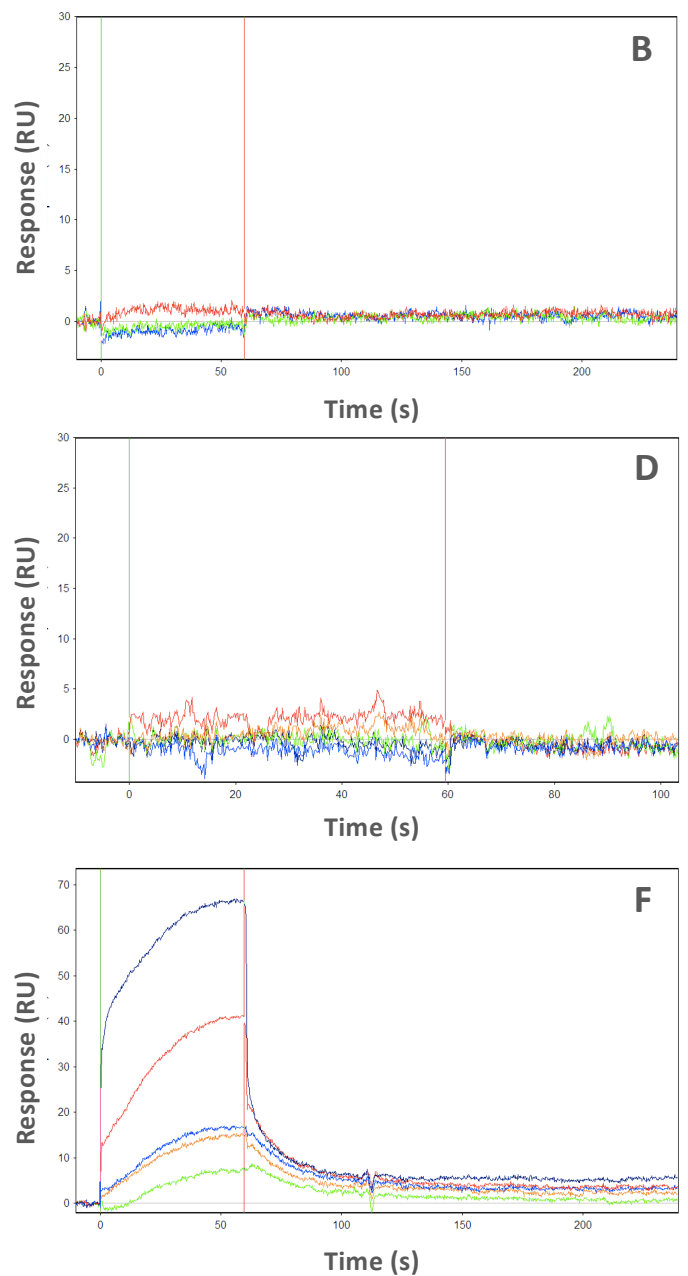

Figure 3. Sensorgrams of competition experiments between the inhibitors CD532 (A), MLN8054 (B), PHA-680626 (C), RPM1722 (D), Alisertib (E), MK8754 (F), ZM447439 (G) and NMyc. The experiment was carried out by injecting N-Myc at concentrations ranging from 125 $\mathrm{nM}$ to $16 \mu \mathrm{M}$. N-Myc was previously diluted in running buffer containing a saturating concentration of the inhibitor (at least 10 times higher than its $\mathrm{Kd}$ ). Apparent $\mathrm{Kd}$ values of $\mathrm{N}$ Myc are shown in Table 2. Apparent Kd values for the negative controls MK8754 and ZM447439 are $690 \mathrm{nM}$ and $4 \mu \mathrm{M}$, respectively. 
Figure 4

A.

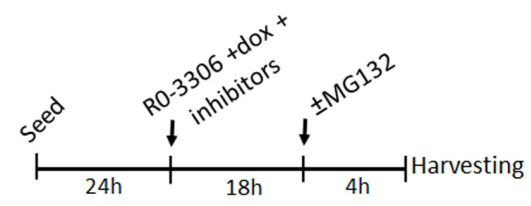

B.

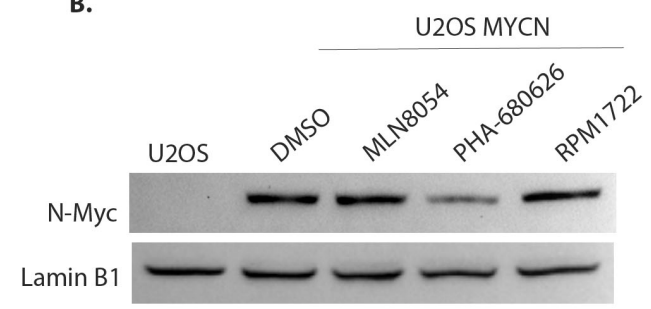

C.
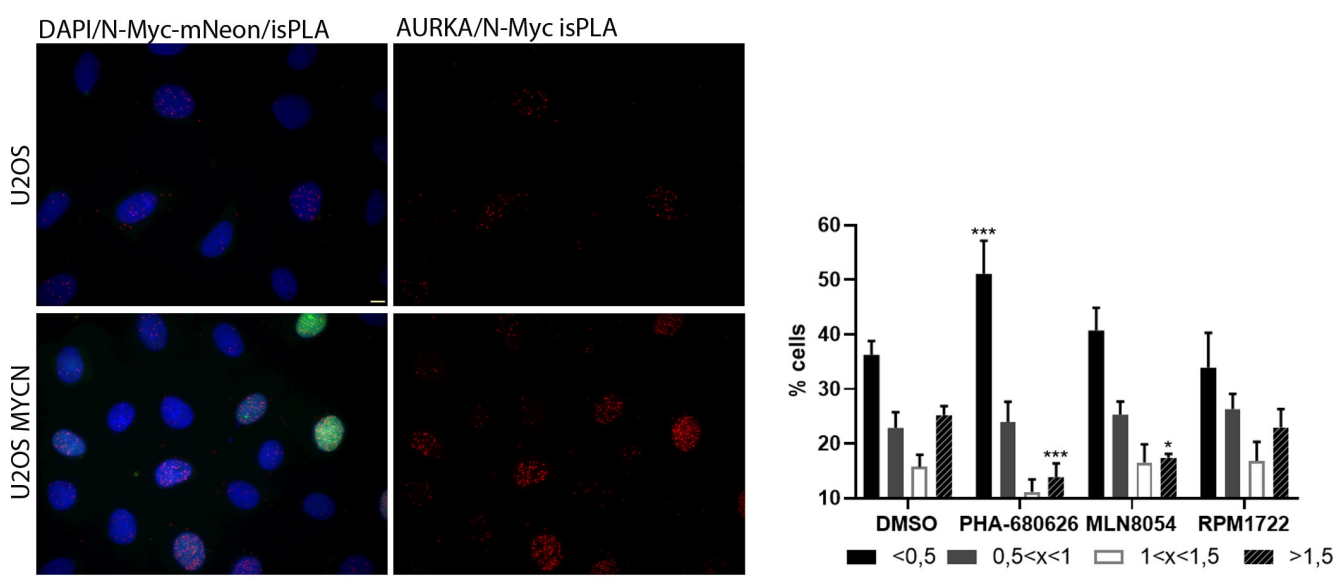

Figure 4. PHA-680626 is able to decrease AURKA/N-Myc interaction. (A) Schematization of the combined synchronization and treatment protocol. (B) Western Blot analysis from U2OS and U2OS MYCN cell lines treated with $1 \mu \mathrm{M}$ of each compound, or DMSO as control, following the protocol described in (A), without MG132. Lamin B1 was used as loading control. (C) in situ Proximity Ligation assays to visualize the AURKA/N-Myc complex formation. The fluorescence panels show examples of the negative (U2OS that do not express N-Myc) and the positive (U2OS MYC dox-induced) reference cultures. The histograms on the right show the distribution (\%) of cells in classes, defined by isPLA spot values. Values represent isPLA spots per nucleus, normalized to the average value in control cells (at least 1400 measured cells per condition, from 4 independent experiments); mean and standard deviation are shown. Statistical analysis by 2 way ANOVA-Tukey's multiple comparison, *: $p<0.05 ; * * *: p<0.001$. Scale bar: $10 \mu \mathrm{m}$. 


\section{Figure 5}

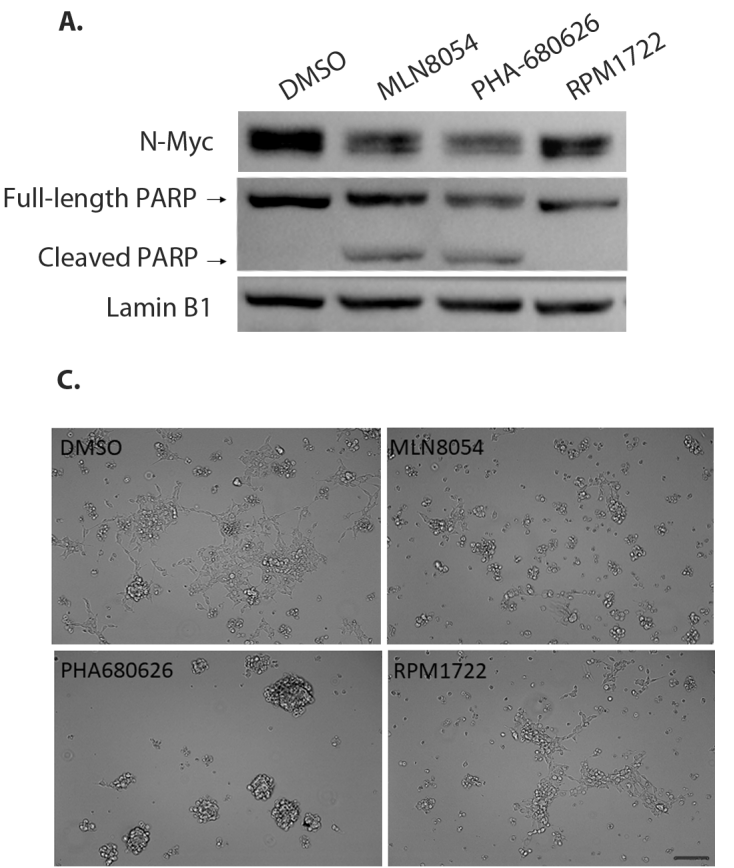

B.
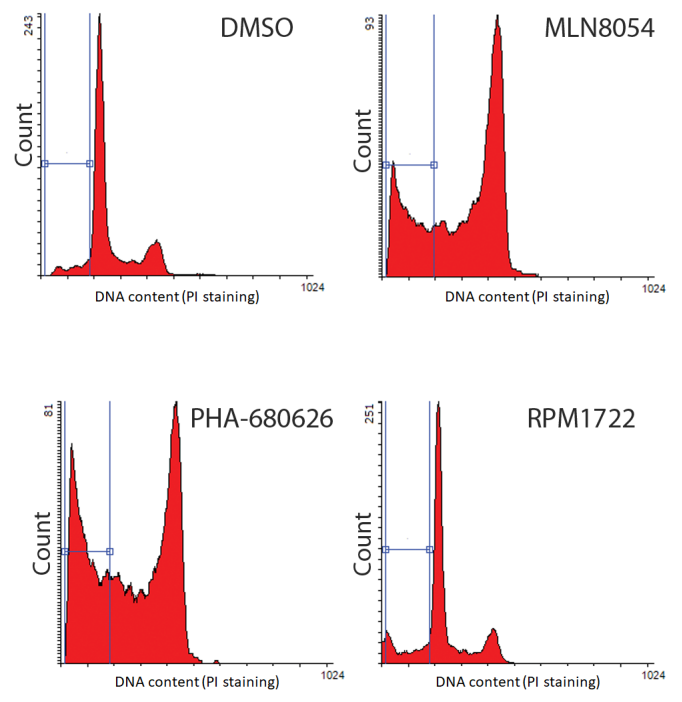

Figure 5. PHA-680626 causes N-Myc protein decrease and cellular stress in neuroblastoma cells. (A) Western Blot analysis from IMR-32 neuroblastoma cell line treated with $1 \mu \mathrm{M}$ of each inhibitor, or DMSO for control, for $48 \mathrm{~h}$. Both full length and cleaved PARP signals are shown, as indicated. (B) FACS analysis of IMR-32 cells treated as in (A) and stained with PI. The region used to evaluate the fraction of cells displaying sub-G1 DNA content is indicated. (C) Morphological appearance of IMR-32 cultures by bright-field microscopy (20x magnification), treated as in (A). Scale bar: $100 \mu \mathrm{m}$. 